\title{
Water-wave scattering by a semi-infinite periodic array of arbitrary bodies
}

\author{
By MALTE A. PETER AND MICHAEL H. MEYLAN \\ ${ }^{1}$ Centre for Industrial Mathematics, FB3, University of Bremen, Germany \\ ${ }^{2}$ Department of Mathematics, University of Auckland, New Zealand \\ mpeter@math.uni-bremen.de; meylan@math.auckland.ac.nz
}

We consider the scattering by a semi-infinite array of bodies of arbitrary geometry excited by an incident wave in the linear water-wave formulation (which reduces to the simpler case of Helmholtz scattering if the depth dependence can be removed). The theory presented here is extremely general, and we present example calculations for a floating elastic plate (a highly non-trivial scatterer). The solution method follows closely from the solution for point scatterers in a medium governed by Helmholtz's equation, presented by Linton \& Martin (2004). We require several extensions to this theory: we consider water-wave scattering, we allow for bodies of arbitrary scattering geometry and we show how to include the effects of bound waves (called Rayleigh-Bloch waves in the water-wave context) in the formulation. We present results for scattering by arrays of cylinders showing the convergence of our methods, and also some results for the case of scattering by floating elastic plates and fixed docks.

\section{Introduction}

Many physical structures (antennas, off-shore platforms etc.) consist of repeating elements which are evenly spaced. In many cases, it is possible to considerably simplify the problem by assuming that the elements are arranged in an infinite array (i.e. the array extends in one dimension to infinity in both directions) and to then solve for the resultant scattering by assuming a periodic relationship between each element of the array. If the forcing is also periodic, this method then reduces the problem to solving for a single element of the array, but it requires the influence of all other elements in the infinite array to be included. The idea of studying infinite arrays is not new and is related to the theory of diffraction gratings. It is not our intention to give a complete summary of the literature, and we reference the excellent review article McIver (2002) which describes both finite and infinite array effects.

The most significant early results for scattering by infinite arrays were those of von Ignatowsky (1914), who considered the problem of scattering by cylinders of arbitrary cross-section in a medium governed by Helmholtz's equation, and of Twersky (1962), who solved for circular cylinders using a separation-of-variables approach. This problem was revisited by Linton \& Evans (1993) where numerical results were obtained, and it was further extended by Porter \& Evans (1999) to the case of non-circular geometries using a Green's function method. Recently, Peter et al. (2006) have solved for an array of bodies of arbitrary geometry in the context of water waves (which reduces to the simpler Helmholtz equation if the depth dependence can be removed). This solution was based on the extension of the interaction theory of Kagemoto \& Yue (1986) developed by Peter \& Meylan (2004). 
The infinite-array solution has been used successfully to predict the motion and forces on large arrays far from the edges. It is natural to consider the more complicated case of a semi-infinite array (one which extends to infinity in only one direction) and to solve this problem so as to be able to model effects near the edge of large finite arrays. This problem is much more challenging than the infinite-array problem both theoretically and numerically, because it is not possible to reduce the solution to an unknown defined on a single scatterer. The problem was considered by Hills \& Karp (1965) and was recently studied by Linton \& Martin (2004), who determined the scattering due to a semi-infinite array composed of infinitely small isotropic scatterers in a two-dimensional medium governed by Helmholtz's equation. Owing to the small-size assumption, they were able to solve the problem using the Wiener-Hopf technique, a method which does not appear to be suitable for extension to more complicated geometries. However, the basic method in Linton \& Martin (2004) (which was to subtract the infinite-array solution) is suitable for extension to arbitrary geometry bodies, as are the expressions for the far field.

There is another important complication in the semi-infinite array problem which is not present in the infinite-array problem. It is well known that some infinite arrays can support bound waves which travel along the array and which do not radiate energy to infinity away from the array. These waves are called Rayleigh-Bloch waves in the waterwave context, and they have other names (guided waves, surface waves) in acoustics and electromagnetics. Generally, they propagate along the array with a dominant wavenumber which is greater than that for freely-propagating waves and they decay exponentially away from the array. It is possible to obtain Rayleigh-Bloch waves which have a dominant wavelength less than the freely propagating waves as was shown by Porter \& Evans (2005) but this occurs only for very special geometries and we will not consider this case here.

Rayleigh-Bloch waves have been constructed for a range of geometries (see the reference in Porter \& Evans 1999) and a proof has been given for their existence in quite general situations by Linton \& McIver (2002). However, all these results have been for a medium governed by Helmholtz's equation, which requires uniform geometry in depth in the water-wave context. No Rayleigh-Bloch waves have been found (numerically or analytically) in the more general water-wave case in which the bodies have some complicated depth dependence, and it remains an open question whether they can exist in this situation. It turns out that for the case of a semi-infinite array subject to an incident wave, Rayleigh-Bloch waves can be excited and, hence, need to be taken into consideration in the derivation of a solution method (this idea was suggested by Richard Porter, personal communication). Including the Rayleigh-Bloch waves in the formulation for the semi-infinite case requires a further extension of the theory of Linton \& Martin (2004).

The solution for a semi-infinite array given here is an extension of the solution for an infinite array given in Peter et al. (2006). An outline of the paper is as follows. Section 2 contains the explicit formulation of the problem and the derivation of a generic system of equations which applies to both the infinite array and the semi-infinite array. In $\S 3$, the infinite-array problem is briefly revisited, since it is required for the solution for the semi-infinite array. The phenomenon of Rayleigh-Bloch waves is discussed in $\S 4$, before two different solution methods for the semi-infinite array are presented in $\S 5$. It should be noted that these two methods only differ in the special (and more complicated) case that a Rayleigh-Bloch wave is excited. For the case when there is no Rayleigh-Bloch wave excited, the two methods coincide. The far-field representation is derived in $\S 6$ and the numerically efficient calculation of some series arising in the problem is discussed in 


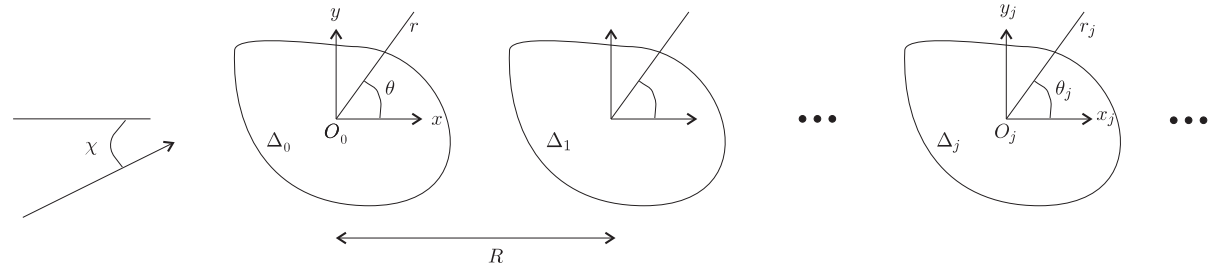

FigURE 1. Plan view of the relation between the bodies.

$\S 7$. Section 8 is concerned with numerical results for floating plates and, in particular, it contains a convergence study for rigid bottom-mounted circular cylinders.

\section{Formulation of the general problem}

The formulation and notation follows closely from Peter et al. (2006) where the solution for an infinite array was presented and we will omit some details in the derivation which can be found in Peter et al. (2006). We consider the water-wave scattering of a plane wave by a semi-infinite array of identical vertically non-overlapping bodies, denoted by $\Delta_{j}$. The mean-centre positions $O_{j}$ of $\Delta_{j}$ are assumed to be $O_{j}=(j R, 0), j=0, \ldots, \infty$, where the distance between the bodies, $R$, is supposed sufficiently large so that there is no intersection of the smallest circular cylinder, which contains each body, with any other body. The ambient plane wave is assumed to travel in the direction $\chi \in(0, \pi)$ where $\chi$ is measured with respect to the $x$-axis. Let $\left(r_{j}, \theta_{j}, z\right)$ be the local cylindrical coordinates of the $j$ th body, $\Delta_{j}$, where the $z$-axis points vertically upwards. Note that the zeroth body is centred at the origin and its local cylindrical coordinates coincide with the global ones, $(r, \theta, z)$. Figure 1 illustrates the setting.

The equations of motion for the water are derived from the linearized inviscid theory. Assuming that the motion is time-harmonic with radian frequency $\omega$, the velocity potential can be expressed as the real part of a complex quantity,

$$
\Phi(\mathbf{x}, t)=\operatorname{Re}\left\{\phi(\mathbf{x}) \mathrm{e}^{-\mathrm{i} \omega t}\right\} .
$$

Writing $\alpha=\omega^{2} / g$ where $g$ is the acceleration due to gravity, the complex water velocity potential $\phi$ has to satisfy the standard boundary-value problem

$$
\begin{aligned}
\nabla^{2} \phi & =0, & \mathrm{x} & \in D, \\
\frac{\partial \phi}{\partial z} & =\alpha \phi, & & \mathbf{x} \in \Gamma^{\mathrm{f}}, \\
\frac{\partial \phi}{\partial z} & =0, & & \mathbf{x} \in D, z=-d,
\end{aligned}
$$

where $D=\left(\mathbb{R}^{2} \times(-d, 0)\right) \backslash \bigcup_{j} \bar{\Delta}_{j}$ is the domain occupied by the water and $\Gamma^{\mathrm{f}}$ is the free water surface. At the immersed body surface $\Gamma_{j}$ of $\Delta_{j}$, the water velocity potential has to equal the normal velocity of the body $\mathbf{v}_{j}$,

$$
\frac{\partial \phi}{\partial \mathbf{n}}=\mathbf{v}_{j}, \quad \mathbf{x} \in \Gamma_{j} .
$$

Moreover, a radiation condition is imposed, ensuring that there are only outgoing waves from each scatterer (more precisely, we only allow for scattered waves which can be represented in the expansion (2.5)). The ambient incident potential is denoted by $\phi^{\text {In }}$ and the positive wavenumber $k$ is related to $\alpha$ by the dispersion relation

$$
\alpha=k \tanh k d,
$$


and the values of $k_{m}, m>0$, are given as positive real roots of the dispersion relation

$$
\alpha+k_{m} \tan k_{m} d=0 .
$$

For ease of notation, we write $k_{0}=-\mathrm{i} k$. Note that $k_{0}$ is a (purely imaginary) root of (2.4).

\subsection{Eigenfunction expansion of the potential}

The scattered potential of a body $\Delta_{j}$ can be expanded in singular cylindrical eigenfunctions,

$$
\phi_{j}^{\mathrm{S}}\left(r_{j}, \theta_{j}, z\right)=\sum_{m=0}^{\infty} f_{m}(z) \sum_{\mu=-\infty}^{\infty} A_{m \mu}^{j} K_{\mu}\left(k_{m} r_{j}\right) \mathrm{e}^{\mathrm{i} \mu \theta_{j}},
$$

with discrete coefficients $A_{m \mu}^{j}$, where $f_{m}$ are the orthonormal vertical eigenfunctions defined by

$$
f_{m}(z)=\frac{\cos k_{m}(z+d)}{\cos k_{m} d} .
$$

In (2.5), the term for $m=0$ corresponds to the propagating modes while the terms for $m \geq 1$ correspond to the decaying modes. The incident potential upon body $\Delta_{j}$ can also be expanded in regular cylindrical eigenfunctions,

$$
\phi_{j}^{\mathrm{I}}\left(r_{j}, \theta_{j}, z\right)=\sum_{n=0}^{\infty} f_{n}(z) \sum_{\nu=-\infty}^{\infty} D_{n \nu}^{j} I_{\nu}\left(k_{n} r_{j}\right) \mathrm{e}^{\mathrm{i} \nu \theta_{j}},
$$

with discrete coefficients $D_{n \nu}^{j}$. In these expansions, $I_{\nu}$ and $K_{\nu}$ denote the modified Bessel function of the first and second kind, respectively, both of order $\nu$ (Abramowitz \& Stegun 1970). For future reference, we remark that, for real $x$,

$$
K_{\nu}(-\mathrm{i} x)=\frac{\pi \mathrm{i}^{\nu+1}}{2} H_{\nu}^{(1)}(x) \quad \text { and } \quad I_{\nu}(-\mathrm{i} x)=\mathrm{i}^{-\nu} J_{\nu}(x)
$$

with $H_{\nu}^{(1)}$ and $J_{\nu}$ denoting the Hankel function and the Bessel function, respectively, both of first kind and order $\nu$ (Abramowitz \& Stegun 1970).

\subsection{Representation of the ambient wavefield in the eigenfunction representation}

In Cartesian coordinates centred at the origin, the ambient plane wavefield is given by

$$
\phi^{\operatorname{In}}(x, y, z)=\frac{A g}{\omega} f_{0}(z) \mathrm{e}^{\mathrm{i} k(x \cos \chi+y \sin \chi)},
$$

where $A$ is the amplitude (in displacement) and $\chi$ is the angle between the $x$-axis and the direction in which the wavefield travels (also cf. figure 1). In the eigenfunction expansion centred at the origin, this can be expressed as

$$
\phi^{\operatorname{In}}(r, \theta, z)=\frac{A g}{\omega} f_{0}(z) \sum_{\nu=-\infty}^{\infty} \mathrm{e}^{\mathrm{i} \nu(\pi / 2-\theta+\chi)} J_{\nu}(k r) .
$$

In order to represent the ambient wavefield, which is incident upon all bodies, in the eigenfunction expansion of an incoming wave in the local coordinates of the body, a phase factor has to be defined,

$$
P_{l}=\mathrm{e}^{\mathrm{i} k l R \cos \chi}
$$


which accounts for the position from the origin. Including this phase factor and making use of (2.8), the ambient wavefield at the $l$ th body is given by

$$
\phi^{\operatorname{In}}\left(r_{l}, \theta_{l}, z\right)=\frac{A g}{\omega} P_{l} f_{0}(z) \sum_{\nu=-\infty}^{\infty} \mathrm{e}^{\mathrm{i} \nu(\pi-\chi)} I_{\nu}\left(k_{0} r_{l}\right) \mathrm{e}^{\mathrm{i} \nu \theta_{l}} .
$$

We can therefore define the coefficients of the ambient wavefield in the eigenfunction expansion of an incident wave,

$$
\tilde{D}_{n \nu}^{l}= \begin{cases}\frac{A g}{\omega} P_{l} \mathrm{e}^{\mathrm{i} \nu(\pi-\chi)}, & n=0 \\ 0, & n>0 .\end{cases}
$$

Note that the decaying coefficients are all zero, due to the propagating nature of the ambient wave.

\subsection{Derivation of the general system of equations}

Making use of a general interaction theory (Kagemoto \& Yue 1986; Peter \& Meylan 2004), a system of equations for the unknown coefficients (in the expansion (2.5)) of the scattered wavefields of all bodies is developed. The methodology, sometimes also referred to as a $T$-matrix method, is based on transforming the scattered potential of $\Delta_{j}$ into an incident potential upon $\Delta_{l}(j \neq l)$. Doing this for all bodies simultaneously, and relating the incident and scattered potential for each body, a system of equations for the unknown coefficients is developed. This system of equations can then be simplified for the two cases of an infinite and a semi-infinite array.

The scattered potential $\phi_{j}^{\mathrm{S}}$ of body $\Delta_{j}$ needs to be represented in terms of the incident potential $\phi_{l}^{\mathrm{I}}$ upon $\Delta_{l}, j \neq l$. From figure 1 we can see that this can be accomplished by using Graf's addition theorem for Bessel functions given in Abramowitz \& Stegun (1970, eq. 9.1.79),

$$
K_{\tau}\left(k_{m} r_{j}\right) \mathrm{e}^{\mathrm{i} \tau\left(\theta_{j}-\varphi_{j-l}\right)}=\sum_{\nu=-\infty}^{\infty} K_{\tau+\nu}\left(k_{m}|j-l| R\right) I_{\nu}\left(k_{m} r_{l}\right) \mathrm{e}^{\mathrm{i} \nu\left(\pi-\theta_{l}+\varphi_{j-l}\right)}, \quad j \neq l,
$$

which is valid provided that $r_{l}<R$. The angles $\varphi_{n}$ account for the difference in direction depending if the $j$ th body is located to the left or to the right of the $l$ th body, and they are defined by

$$
\varphi_{n}= \begin{cases}\pi, & n>0 \\ 0, & n<0\end{cases}
$$

The limitation $r_{l}<R$ only requires that the escribed cylinder of each body $\Delta_{l}$ does not enclose any other origin $O_{j}(j \neq l)$. However, the expansion of the scattered and incident potential in cylindrical eigenfunctions is only valid outside the escribed cylinder of each body. Therefore the condition that the escribed cylinder of each body $\Delta_{l}$ does not enclose any other origin $O_{j}(j \neq l)$ is superseded by the more stringent restriction that the escribed cylinder of each body may not contain any other body.

Making use of the eigenfunction expansion as well as equation (2.10), the scattered potential of $\Delta_{j}$ (cf. (2.5)) can be expressed in terms of the incident potential upon $\Delta_{l}$ as

$$
\begin{aligned}
& \phi_{j}^{\mathrm{S}}\left(r_{l}, \theta_{l}, z\right) \\
& \quad=\sum_{m=0}^{\infty} f_{m}(z) \sum_{\nu=-\infty}^{\infty}\left[\sum_{\tau=-\infty}^{\infty} A_{m \tau}^{j}(-1)^{\nu} K_{\tau-\nu}\left(k_{m}|j-l| R\right) \mathrm{e}^{\mathrm{i}(\tau-\nu) \varphi_{j-l}}\right] I_{\nu}\left(k_{m} r_{l}\right) \mathrm{e}^{\mathrm{i} \nu \theta_{l}} .
\end{aligned}
$$


The ambient incident wavefield $\phi^{\text {In }}$ can also be expanded in the eigenfunctions corresponding to the incident wavefield upon $\Delta_{l}$. Let $\tilde{D}_{n \nu}^{l}$ denote the coefficients of this ambient incident wavefield in the incoming eigenfunction expansion for $\Delta_{l}$ (cf. §2.2). The total incident wavefield upon body $\Delta_{j}$ can now be expressed as

$$
\begin{aligned}
\phi_{l}^{\mathrm{I}}\left(r_{l}, \theta_{l}, z\right)= & \phi^{\mathrm{In}}\left(r_{l}, \theta_{l}, z\right)+\sum_{j \in \mathcal{X}_{l}} \phi_{j}^{\mathrm{S}}\left(r_{l}, \theta_{l}, z\right) \\
= & \sum_{n=0}^{\infty} f_{n}(z) \sum_{\nu=-\infty}^{\infty}\left[\tilde{D}_{n \nu}^{l}+\sum_{j \in \mathcal{X}_{l}} \sum_{\tau=-\infty}^{\infty} A_{n \tau}^{j}(-1)^{\nu} K_{\tau-\nu}\left(k_{n}|j-l| R\right) \mathrm{e}^{\mathrm{i}(\tau-\nu) \varphi_{j-l}}\right] \\
& \times I_{\nu}\left(k_{n} r_{l}\right) \mathrm{e}^{\mathrm{i} \nu \theta_{l}},
\end{aligned}
$$

where $\mathcal{X}=\mathbb{Z}$ (the set of all integers) in the infinite-array case and $\mathcal{X}=\mathbb{N}$ (the set of natural numbers which, in particular, includes zero) for the semi-infinite array and $\mathcal{X}_{l}=\mathcal{X} \backslash\{l\}$. The coefficients of the total incident potential upon $\Delta_{l}$ are therefore given by

$$
D_{n \nu}^{l}=\tilde{D}_{n \nu}^{l}+\sum_{j \in \mathcal{X}_{l}} \sum_{\tau=-\infty}^{\infty} A_{n \tau}^{j}(-1)^{\nu} K_{\tau-\nu}\left(k_{n}|j-l| R\right) \mathrm{e}^{\mathrm{i}(\tau-\nu) \varphi_{j-l}}
$$

$n \in \mathbb{N}, \nu \in \mathbb{Z}, l \in \mathcal{X}$.

In general, it is possible to relate the total incident and scattered partial waves for any body through the diffraction characteristics of that body in isolation. There exist diffraction transfer operators $B^{l}$, sometimes also referred to as $T$-matrices, that relate the coefficients of the incident and scattered partial waves, such that

$$
A_{m \mu}^{l}=\sum_{n=0}^{\infty} \sum_{\nu=-\infty}^{\infty} B_{m n \mu \nu}^{l} D_{n \nu}^{l}
$$

where $A^{l}$ are the scattered modes due to the incident modes $D^{l}$. Note that since it is assumed that all bodies are identical in this setting, only one diffraction transfer operator, $B$, is required. Assuming that the diffraction transfer operator is known, the substitution of (2.11) into (2.12) gives the required equations to determine the coefficients of the scattered wavefields of all bodies,

$$
A_{m \mu}^{l}=\sum_{n=0}^{\infty} \sum_{\nu=-\infty}^{\infty} B_{m n \mu \nu}^{l}\left[\tilde{D}_{n \nu}^{l}+\sum_{j \in \mathcal{X}_{l}} \sum_{\tau=-\infty}^{\infty} A_{n \tau}^{j}(-1)^{\nu} K_{\tau-\nu}\left(k_{n}|j-l| R\right) \mathrm{e}^{\mathrm{i}(\tau-\nu) \varphi_{j-l}}\right],
$$

$m \in \mathbb{N}, \mu \in \mathbb{Z}, l \in \mathcal{X}$.

\section{The infinite array}

For the infinite array, we have $\mathcal{X}=\mathbb{Z}$. Due to the periodicity of the geometry and of the incident wave, the coefficients $A_{m \mu}^{l}$ can be written as $A_{m \mu}^{l}=P_{l} A_{m \mu}^{0}=P_{l} A_{m \mu}$, say. The same can be done for the coefficients of the incident ambient wave, i.e. $\tilde{D}_{n \nu}^{l}=P_{l} \tilde{D}_{n \nu}$ (also cf. §2.2). Noting that $P_{l}^{-1}=P_{-l}$ and $P_{j} P_{l}=P_{j+l},(2.13)$ simplifies to

$$
A_{m \mu}=\sum_{n=0}^{\infty} \sum_{\nu=-\infty}^{\infty} B_{m n \mu \nu}\left[\tilde{D}_{n \nu}+(-1)^{\nu} \sum_{\tau=-\infty}^{\infty} A_{n \tau} \sum_{\substack{j=-\infty \\ j \neq 0}}^{\infty} P_{j} K_{\tau-\nu}\left(k_{n}|j| R\right) \mathrm{e}^{\mathrm{i}(\tau-\nu) \varphi_{j}}\right] .
$$


Introducing the constants

$$
\sigma_{\nu}^{n}=\sum_{\substack{j=-\infty \\ j \neq 0}}^{\infty} P_{j} K_{\nu}\left(k_{n}|j| R\right) \mathrm{e}^{\mathrm{i} \nu \varphi_{j}}=\sum_{j=1}^{\infty}\left(P_{-j}+(-1)^{\nu} P_{j}\right) K_{\nu}\left(k_{n} j R\right),
$$

which can be evaluated separately since they do not contain any unknowns, the problem reduces to

$$
A_{m \mu}=\sum_{n=0}^{\infty} \sum_{\nu=-\infty}^{\infty} B_{m n \mu \nu}\left[\tilde{D}_{n \nu}+(-1)^{\nu} \sum_{\tau=-\infty}^{\infty} A_{n \tau} \sigma_{\tau-\nu}^{n}\right] .
$$

The efficient computation of the constants $\sigma_{\nu}^{n}$ is discussed in $\S 7$.

For numerical calculations, the infinite sums in (3.3) have to be truncated. Implying a suitable truncation, the diffraction transfer operator can be represented by a matrix B, the finite-depth diffraction transfer matrix. Truncating the coefficients accordingly, defining $\mathbf{a}^{\text {inf }}$ to be the vector of the coefficients of the scattered potential, $\mathbf{d}^{\text {In }}$ to be the vector of coefficients of the ambient wavefield, and making use of a coordinatetransformation matrix $\mathbf{S}^{\text {inf }}$ given by

$$
\left(\mathbf{S}^{\mathrm{inf}}\right)_{p q}=(-1)^{q} \sigma_{p-q}^{n},
$$

a linear system of equations for the unknown coefficients follows from equations (3.3),

$$
\left(\mathbf{I}-\mathbf{B}{ }^{\mathrm{t}} \mathbf{S}^{\mathrm{inf}}\right) \mathbf{a}^{\mathrm{inf}}=\mathbf{B} \mathbf{d}^{\mathrm{In}},
$$

where the left superscript $t$ indicates transposition and $\mathbf{I}$ is the identity matrix of the same dimension as $\mathbf{B}$.

\section{Rayleigh-Bloch waves}

Before turning to the derivation of the system of equations for the semi-infinite array, we need to discuss a special type of surface wave, so called Rayleigh-Bloch waves. Rayleigh-Bloch waves are waves which travel along an infinite periodic array and which decay exponentially away from the array. Only in very exceptional cases (which will not be considered here) are they excited by plane incident waves (Porter \& Evans 2005). Rayleigh-Bloch waves (if they can be supported by the structure) will be excited in a semi-infinite array provided that $k<\pi / R$.

Rayleigh-Bloch waves are observed for a very general class of arrays, where the medium is governed by the two-dimensional Helmholtz equation (Linton \& McIver 2002). In the water-wave context this means that the structures must have a depth dependence which can be removed, so that the problem reduces to two dimensions. In the more general water-wave context, no Rayleigh-Bloch waves have been found. It seems likely that such waves will exist only for very special geometries and frequencies, but this remains only a conjecture at the present time. There are many off-shore structures which do support Rayleigh-Bloch waves, e.g. bottom-mounted cylinders. We also assume that only a single Rayleigh-Bloch wave exists for a given frequency. Some more information on RayleighBloch waves can be found in Porter \& Evans (1999); Linton \& McIver (2002).

For the problem under consideration, it is important to see whether the structure supports a Rayleigh-Bloch wave because, in this case, we need to give special attention in the subsequent derivations. The Rayleigh-Bloch wavenumber $\beta$ is the value for which the operator $-\nabla^{2}$, subject to the periodicity conditions

$$
\left.\phi\right|_{x=0}=\left.\mathrm{e}^{\mathrm{i} R \beta} \phi\right|_{x=R},\left.\quad \partial_{x} \phi\right|_{x=0}=\left.\mathrm{e}^{\mathrm{i} R \beta} \partial_{x} \phi\right|_{x=R}
$$


and appropriate boundary conditions at the body surfaces, has an eigenvalue in the interval $(k, \pi / R]$. This corresponds to a wave travelling down the array with phase factor

$$
Q_{j}=\mathrm{e}^{\mathrm{i} j R \beta} .
$$

Numerically, the Rayleigh-Bloch wavenumber is easy to determine: In terms of the matrices of the truncated problem, this is the value of $\beta$ for which

$$
\operatorname{det}\left(\mathbf{I}-\mathbf{B}{ }^{\mathrm{t}} \mathbf{S}^{\inf }(\beta)\right)=0
$$

where $P_{j}$ has been replaced by $Q_{j}$ in $(3.2)$ and we have written $\mathbf{S}^{\inf }(\beta)$ to emphasize the dependence of $\mathbf{S}^{\text {inf }}$ on $\beta$ (note that $\beta=k \cos \chi$ in $\S 3$ ).

For future reference, we denote the Rayleigh-Bloch wavenumber by $\beta$ and the corresponding eigenvector by $v$ (i.e. $v$ is the eigenvector of the non-truncated operator in (4.3) associated with the eigenvalue zero), which has been normalized by $\left(\sum_{m, \mu} v_{m \mu}^{2}\right)^{\frac{1}{2}}$.

\section{The semi-infinite array}

In this section, we present two different methods for determining the solution of the semi-infinite-array problem. These two methods only differ if a Rayleigh-Bloch wave is present; they coincide in the no-Rayleigh-Bloch-wave case. Note that the coefficients of the Rayleigh-Bloch wave in the eigenfunction expansion (2.5) are given by a constant multiple, $c$, of the eigenvector $v$ and that $v$ can be determined a-priori (cf. $\S 4$ ).

The idea of the first method is to derive a system of equations for the difference between the coefficients of the semi-infinite array and that of the infinite array and the Rayleigh-Bloch wave. Assuming that this difference must approach zero as the number of the body becomes large, the constant $c$ can be determined (by a least-squares fit) as that constant which realizes this condition. The second method makes use of the idea that far down the array, the difference of the coefficients of the semi-infinite and the infinite array should only consist of the Rayleigh-Bloch contribution. This can be used to formulate a system of equations in which the Rayleigh-Bloch wave is completely captured, although a truncation is performed for numerical calculations.

\subsection{Non-Rayleigh-Bloch case or (if present) subtraction of the Rayleigh-Bloch wave}

In order to avoid confusion, from now on, we denote the coefficients of the semi-infinite array by $X_{m \mu}^{j}$. Note that $j \in \mathcal{X}=\mathbb{N}$, in this case. As $j$ becomes large, it can be expected that the coefficients of the infinite array and the semi-infinite array become more and more similar. However, this is untrue if a Rayleigh-Bloch wave is excited. Therefore, we look for the difference of the coefficients of the semi-infinite array from the infinite array and a Rayleigh-Bloch wave,

$$
Z_{m \mu}^{j}=X_{m \mu}^{j}-P_{j} A_{m \mu}-Q_{j} \mathcal{A}_{m \mu}
$$

where $Q_{j}$ is the phase factor of the Rayleigh-Bloch wave (cf. (4.2)), and $\mathcal{A}_{m \mu}$ are the coefficients of the Rayleigh-Bloch wave in the eigenfunction expansion (2.5). When we are in a setting where no Rayleigh-Bloch waves are excited, we just have $\mathcal{A}_{m \mu}=0$. For this, it is sufficient that $k \geq \pi / R$ or that the structure does not support RayleighBloch waves. It has to be noted that the $\mathcal{A}_{m \mu}$ involve an a-priori unknown coefficient the amplitude of the Rayleigh-Bloch wave. A method for the determination of $\mathcal{A}_{m \mu}$ is discussed in $§ 5.2$.

Instead of solving for the $X_{m \mu}^{j}$ directly, we derive a systems of equations for the differences $Z_{m \mu}^{j}$ and solve for those. The system of equations for the coefficients $X_{m \mu}^{j}$ is given 
by (2.13) with $\mathcal{X}=\mathbb{N}$. Using (5.1), a system of equations for $Z_{m \mu}^{j}$ is obtained,

$$
\begin{aligned}
& Z_{m \mu}^{l}+P_{l} A_{m \mu}+Q_{l} \mathcal{A}_{m \mu}=\sum_{n=0}^{\infty} \sum_{\nu=-\infty}^{\infty} B_{m n \mu \nu} \\
& \quad \times\left[\tilde{D}_{n \nu}^{l}+\sum_{j \in \mathbb{N}_{l}} \sum_{\tau=-\infty}^{\infty}\left(Z_{n \tau}^{j}+P_{j} A_{n \tau}+Q_{j} \mathcal{A}_{n \tau}\right)(-1)^{\nu} K_{\tau-\nu}\left(k_{n}|j-l| R\right) \mathrm{e}^{\mathrm{i}(\tau-\nu) \varphi_{j-l}}\right] .
\end{aligned}
$$

Utilizing (3.1), noting that $\mathcal{A}_{m \mu}$ also satisfies (3.1) where $\tilde{D}_{n \nu}=0$ and $P_{j-l}$ has been replaced by $Q_{j-l}$, changing the summation and noting that $\varphi_{n}=0$ for $n<0$, we are left with

$$
\begin{aligned}
Z_{m \mu}^{l}=\sum_{n=0}^{\infty} \sum_{\nu=-\infty}^{\infty} B_{m n \mu \nu}[ & \sum_{j \in \mathbb{N}_{l}} \sum_{\tau=-\infty}^{\infty} Z_{n \tau}^{j}(-1)^{\nu} K_{\tau-\nu}\left(k_{n}|j-l| R\right) \mathrm{e}^{\mathrm{i}(\tau-\nu) \varphi_{j-l}} \\
& -(-1)^{\nu} Q_{l} \sum_{\tau=-\infty}^{\infty} \mathcal{A}_{n \tau} \sum_{j=l+1}^{\infty} Q_{-j} K_{\tau-\nu}\left(k_{n} j R\right) \\
& \left.-(-1)^{\nu} P_{l} \sum_{\tau=-\infty}^{\infty} A_{n \tau} \sum_{j=l+1}^{\infty} P_{-j} K_{\tau-\nu}\left(k_{n} j R\right)\right] .
\end{aligned}
$$

Introducing the constants $\tilde{\varsigma}_{\nu}^{n l}$ and $\hat{\varsigma}_{\nu}^{n l}$ defined by

$$
\tilde{\varsigma}_{\nu}^{n l}=\sum_{j=l+1}^{\infty} P_{-j} K_{\nu}\left(k_{n} j R\right), \quad \hat{\varsigma}_{\nu}^{n l}=\sum_{\substack{j=-l \\ j \neq 0}}^{\infty} Q_{-j} K_{\nu}\left(k_{n} j R\right),
$$

the system of equations simplifies to

$$
\begin{aligned}
Z_{m \mu}^{l}=\sum_{n=0}^{\infty} \sum_{\nu=-\infty}^{\infty} B_{m n \mu \nu} & {\left[\sum_{j \in \mathbb{N}_{l}} \sum_{\tau=-\infty}^{\infty} Z_{n \tau}^{j}(-1)^{\nu} K_{\tau-\nu}\left(k_{n}|j-l| R\right) \mathrm{e}^{\mathrm{i}(\tau-\nu) \varphi_{j-l}}\right.} \\
& \left.-(-1)^{\nu} Q_{l} \sum_{\tau=-\infty}^{\infty} \mathcal{A}_{n \tau} \hat{\kappa}_{\tau-\nu}^{n l}-(-1)^{\nu} P_{l} \sum_{\tau=-\infty}^{\infty} A_{n \tau} \tilde{\zeta}_{\tau-\nu}^{n l}\right] .
\end{aligned}
$$

The efficient computation of the $\tilde{\varsigma}_{\nu}^{n l}$ and $\hat{\varsigma}_{\nu}^{n l}$ is outlined in $\S 7$.

Truncating the coefficients in the same way as for the infinite array, i.e. defining $\mathbf{z}^{l}$ to be the vector of the differences of the coefficients of the scattered potential of the semi-infinite array and the infinite array (denoted by $\mathbf{a}^{\text {inf }}$ ) as well as the Rayleigh-Bloch wave (denoted by $\mathbf{a}^{\mathrm{RB}}$ ) and making use of a coordinate-transformation matrix $\mathbf{T}_{j l}$ given by

$$
\left(\mathbf{T}_{j l}\right)_{p q}=(-1)^{q} K_{p-q}\left(k_{m}|j-l| R\right) \mathrm{e}^{\mathrm{i}(p-q) \varphi_{j-l}}
$$

as well as matrices $\tilde{\mathbf{S}}$ and $\hat{\mathbf{S}}$ mapping the coefficients of the infinite array and the RayleighBloch wave appropriately, respectively,

$$
\left(\tilde{\mathbf{S}}_{l}\right)_{p q}=(-1)^{q} P_{l} \tilde{\varsigma}_{p-q}^{n l}, \quad\left(\hat{\mathbf{S}}_{l}\right)_{p q}=(-1)^{q} Q_{l} \hat{\varsigma}_{p-q}^{n l},
$$

a linear system of equations for the unknown coefficients follows from equations (5.3),

$$
\mathbf{z}_{l}=\mathbf{B}\left(\sum_{\substack{j=0 \\ j \neq l}}^{\infty}{ }^{\mathrm{t}} \mathbf{T}_{j l} \mathbf{z}_{j}+{ }^{\mathrm{t}} \hat{\mathbf{S}}_{l} \mathbf{a}^{\mathrm{RB}}-{ }^{\mathrm{t}} \tilde{\mathbf{S}}_{l} \mathbf{a}^{\mathrm{inf}}\right), \quad l=0, \ldots, \infty .
$$

It is worth noting that this method also provides a means of determining whether a 
Rayleigh-Bloch wave is present or not. If (5.6) is solved with $\mathbf{a}^{\mathrm{RB}}=\mathbf{0}$ and the $\mathbf{z}_{l}$ do not decay to zero for large $l$, a Rayleigh-Bloch wave must be existent. Nevertheless, it appears that the method for determining the presence of a Rayleigh-Bloch wave based on the infinite-array solution of Peter et al. (2006) described in $\S 4$ is more direct. Moreover, if a Rayleigh-Bloch wave is present, the method of $\S 4$ automatically supplies its wavenumber.

\subsection{Determination of $c$}

The structure of the amplitude of the Rayleigh-Bloch wave is $\mathcal{A}=c v$ with a complex constant $c$. Since the eigenvector $v$ can be determined from (4.3), the problem reduces to finding the constant $c$. In what follows, we suggest a way for its numerical approximation using a least-squares approach.

One approach for the approximation of $\mathbf{a}^{\mathrm{RB}}$ is to use a least-squares fit. Substituting $\mathbf{a}^{\mathrm{RB}}=c \mathbf{v}$ in (5.6), the system of equations with $N$ bodies in the calculation reads

$$
\begin{aligned}
{\left[\begin{array}{cccc}
\mathbf{I} & -\mathbf{B}^{\mathrm{t}} \mathbf{T}_{21} & \ldots & -\mathbf{B}^{\mathrm{t}} \mathbf{T}_{N 1} \\
-\mathbf{B}^{\mathrm{t}} \mathbf{T}_{12} & \mathbf{I} & \ldots & -\mathbf{B}^{\mathrm{t}} \mathbf{T}_{N 2} \\
\vdots & & \ddots & \vdots \\
-\mathbf{B}^{\mathrm{t}} \mathbf{T}_{1 N} & \ldots & & \mathbf{I}
\end{array}\right]\left[\begin{array}{c}
\mathbf{z}_{1} \\
\mathbf{z}_{2} \\
\vdots \\
\mathbf{z}_{N}
\end{array}\right] } \\
=c\left[\begin{array}{c}
\mathbf{B}^{\mathrm{t}} \hat{\mathbf{S}}_{1} \mathbf{v} \\
\mathbf{B}^{\mathrm{t}} \hat{\mathbf{S}}_{2} \mathbf{v} \\
\vdots \\
\mathbf{B}^{\mathrm{t}} \hat{\mathbf{S}}_{N} \mathbf{v}
\end{array}\right]-\left[\begin{array}{c}
\mathbf{B}^{\mathrm{t}} \tilde{\mathbf{S}}_{1} \mathbf{a}^{\mathrm{inf}} \\
\mathbf{B}^{\mathrm{t}} \tilde{\mathbf{S}}_{2} \mathbf{a}^{\mathrm{inf}} \\
\vdots \\
\mathbf{B}^{\mathrm{t}} \tilde{\mathbf{S}}_{N} \mathbf{a}^{\mathrm{inf}}
\end{array}\right],
\end{aligned}
$$

where $\mathbf{I}$ denotes the identity matrix which is of the same dimension as $\mathbf{B}$. This can be written as $\mathbf{A z}=c \mathbf{b}-\mathbf{c}$.

Using the fact that the $\mathbf{z}_{j}$ should decay to zero for increasing $j$ if the Rayleigh-Bloch wave is subtracted correctly, $c$ is given as the least-squares solution of the over-determined system

$$
c \mathbf{A}^{-1} \mathbf{b}-\mathbf{A}^{-1} \mathbf{c}=0 .
$$

Since only the $\mathbf{z}_{N}$ (or the $\mathbf{z}_{j}$ of the last few bodies) should be close to zero, only the last rows of (5.8) should be used for the least-squares fit.

\subsection{Projection method}

If a sufficient number of bodies is used in the semi-infinite-array calculation, $N$ say, it is reasonable to assume that the coefficients of the last body $Z^{N}$ contain only the RayleighBloch wave. For this purpose, consider the system of equations without subtraction of the Rayleigh-Bloch wave. This corresponds to (5.3) with $\mathcal{A}_{m \mu}=0$,

$$
\begin{aligned}
Z_{m \mu}^{l}=\sum_{n=0}^{\infty} \sum_{\nu=-\infty}^{\infty} B_{m n \mu \nu}\left[\sum_{j \in \mathbb{N}_{l}} \sum_{\tau=-\infty}^{\infty} Z_{n \tau}^{j}(-1)^{\nu} K_{\tau-\nu}\left(k_{n}|j-l| R\right) \mathrm{e}^{\mathrm{i}(\tau-\nu) \varphi_{j-l}}\right. & \left.-(-1)^{\nu} P_{l} \sum_{\tau=-\infty}^{\infty} A_{n \tau} \tilde{\varsigma}_{\tau-\nu}^{n l}\right] .
\end{aligned}
$$


Since we have to truncate the sum over $j$ for numerical calculations anyway, we split it up as follows. For some sufficiently large number $N$, we can write

$$
\begin{aligned}
Z_{m \mu}^{l} & =\sum_{n=0}^{\infty} \sum_{\nu=-\infty}^{\infty} B_{m n \mu \nu}\left[\sum_{\substack{j=0 \\
j \neq l}}^{N} \sum_{\tau=-\infty}^{\infty} Z_{n \tau}^{j}(-1)^{\nu} K_{\tau-\nu}\left(k_{n}|j-l| R\right) \mathrm{e}^{\mathrm{i}(\tau-\nu) \varphi_{j-l}}\right. \\
& \left.+\sum_{j=N+1}^{\infty} \sum_{\tau=-\infty}^{\infty} Z_{n \tau}^{j}(-1)^{\nu} K_{\tau-\nu}\left(k_{n}|j-l| R\right) \mathrm{e}^{\mathrm{i}(\tau-\nu) \varphi_{j-l}}-(-1)^{\nu} P_{l} \sum_{\tau=-\infty}^{\infty} A_{n \tau} \tilde{\varsigma}_{\tau-\nu}^{n l}\right] .
\end{aligned}
$$

We then assume that the coefficients of the last body $Z^{N}$ contain only the RayleighBloch wave. Therefore, the coefficients $Z_{n \tau}^{j}, j \geq N+1$, can be replaced by the projections of $Z_{m \mu}^{N}$ onto the eigenvector $v_{n \tau}$ multiplied by the Rayleigh-Bloch phase factor $Q_{j-N}$,

$$
Z_{n \tau}^{j}=Q_{j-N} \sum_{m=0}^{\infty} \sum_{\mu=-\infty}^{\infty} Z_{m \mu}^{N} \bar{v}_{m \mu} v_{n \tau}, \quad j \geq N+1,
$$

where the overbar denotes complex conjugation. Note that the double sum is just the scalar product of $Z^{N}$ with $v$. Writing

$$
\mathcal{A}_{n \tau}^{\mathrm{P}}=\sum_{m=0}^{\infty} \sum_{\mu=-\infty}^{\infty} Z_{m \mu}^{N} \bar{v}_{m \mu} v_{n \tau}
$$

for ease of notation, the system then simplifies to

$$
\begin{aligned}
& Z_{m \mu}^{l}=\sum_{n=0}^{\infty} \sum_{\nu=-\infty}^{\infty} B_{m n \mu \nu}\left[\sum_{\substack{j=0 \\
j \neq l}}^{N} \sum_{\tau=-\infty}^{\infty} Z_{n \tau}^{j}(-1)^{\nu} K_{\tau-\nu}\left(k_{n}|j-l| R\right) \mathrm{e}^{\mathrm{i}(\tau-\nu) \varphi_{j-l}}\right. \\
& \left.+\sum_{\tau=-\infty}^{\infty}(-1)^{\nu} \mathcal{A}_{n \tau}^{\mathrm{P}} Q_{l-N} \sum_{j=N+1-l}^{\infty} Q_{j} K_{\tau-\nu}\left(k_{n}|j| R\right) \mathrm{e}^{\mathrm{i}(\tau-\nu) \varphi_{j}}-(-1)^{\nu} P_{l} \sum_{\tau=-\infty}^{\infty} A_{n \tau} \tilde{\varsigma}_{\tau-\nu}^{n l}\right],
\end{aligned}
$$

Introducing yet another sum,

$$
\varsigma_{\nu}^{n l}=\sum_{j=N-l+1}^{\infty} Q_{j} K_{\nu}\left(k_{n} j R\right) \mathrm{e}^{\mathrm{i} \nu \varphi_{j}}
$$

we are left with

$$
\begin{aligned}
Z_{m \mu}^{l}=\sum_{n=0}^{\infty} \sum_{\nu=-\infty}^{\infty} B_{m n \mu \nu} & {\left[\sum_{\substack{j=0 \\
j \neq l}}^{N} \sum_{\tau=-\infty}^{\infty} Z_{n \tau}^{j}(-1)^{\nu} K_{\tau-\nu}\left(k_{n}|j-l| R\right) \mathrm{e}^{\mathrm{i}(\tau-\nu) \varphi_{j-l}}\right.} \\
+ & \left.\sum_{\tau=-\infty}^{\infty}(-1)^{\nu} Q_{l-N} \varsigma_{\tau-\nu}^{n l} \mathcal{A}_{n \tau}^{\mathrm{P}}-(-1)^{\nu} P_{l} \sum_{\tau=-\infty}^{\infty} A_{n \tau} \tilde{\varsigma}_{\tau-\nu}^{n l}\right] .
\end{aligned}
$$

Note that in this approach, the $Z_{m \mu}^{j}$ do not approach zero for large $j$ since they contain the Rayleigh-Bloch wave.

For numerical calculations, the infinite sums in (5.10) have to be truncated. With the same truncation as before (cf. $\S \S 3$ and 5.1), and introducing the matrix $\mathbf{S}$ extending the Rayleigh-Bloch wave to the rest of the array appropriately,

$$
\left(\mathbf{S}_{l}\right)_{p q}=(-1)^{q} Q_{l-N} \varsigma_{p-q}^{n l},
$$

as well as the matrix $\mathbf{V}=\mathbf{v}^{t} \overline{\mathbf{v}}$ (i.e. the dyadic product), a linear system of equations for 
the unknown coefficients follows from equations (5.10),

$$
\mathbf{z}_{l}=\mathbf{B}\left(\sum_{\substack{j=0 \\ j \neq l}}^{N}{ }^{\mathrm{t}} \mathbf{T}_{j l} \mathbf{z}_{j}+{ }^{\mathrm{t}} \mathbf{S}_{l} \mathbf{V} \mathbf{z}_{N}-{ }^{\mathrm{t}} \tilde{\mathbf{S}}_{l} \mathbf{a}^{\mathrm{inf}}\right), \quad l=0, \ldots, N .
$$

Note that if we formally set $v=0$ (i.e. no Rayleigh-Bloch wave is present), this system of equations is the same as that of the (truncated) subtraction method (5.6) without the Rayleigh-Bloch wave.

\section{The far field}

In this section, the far field is examined which describes the scattering far away from the array. First, we define the scattering angles which give the directions of propagation of plane scattered waves far away from the array. Letting $p=2 \pi / R$, define the scattering angles $\chi_{m}$ by

$$
\chi_{m}=\arccos \left(\psi_{m} / k\right) \quad \text { where } \quad \psi_{m}=k \cos \chi+m p
$$

and write $\psi$ for $\psi_{0}$. Also note that $\chi_{0}=\chi$ by definition. If $\left|\psi_{m}\right|<k$, i.e. if

$$
-1<\cos \chi+\frac{m p}{k}<1
$$

we say that $m \in \mathcal{M}$ and then $0<\chi_{m}<\pi$. It turns out (see below) that these angles $\left( \pm \chi_{m}\right.$ for $\left.m \in \mathcal{M}\right)$ are the directions in which plane waves propagate away from the array. If $\left|\psi_{m}\right|>k$ then $\chi_{m}$ is no longer real and the appropriate branch of the arccos function is given by

$$
\arccos t= \begin{cases}\mathrm{i} \operatorname{arccosh} t, & t>1 \\ \pi-\mathrm{i} \operatorname{arccosh}(-t), & t<-1\end{cases}
$$

with $\operatorname{arccosh} t=\log \left(t+\sqrt{t^{2}-1}\right)$ for $t>1$.

\subsection{Infinite array}

The details of the derivation of the far field of the infinite array can be found in Peter et al. (2006) which is an adaptation of the original derivation of Twersky (1962). For the total potential we have

$$
\begin{aligned}
\phi & =\phi^{\mathrm{In}}+\sum_{m=0}^{\infty} f_{m}(z) \sum_{j=-\infty}^{\infty} P_{j} \sum_{\mu=-\infty}^{\infty} A_{m \mu} K_{\mu}\left(k_{m} r_{j}\right) \mathrm{e}^{\mathrm{i} \mu \theta_{j}} \\
& \sim \phi^{\mathrm{In}}+\frac{\pi}{2} f_{0}(z) \sum_{j=-\infty}^{\infty} P_{j} \sum_{\mu=-\infty}^{\infty} A_{0 \mu} \mathrm{i}^{\mu+1} H_{\mu}^{(1)}\left(k r_{j}\right) \mathrm{e}^{\mathrm{i} \mu \theta_{j}},
\end{aligned}
$$

as $k r \rightarrow \infty$, away from the array axis $y=0$, where we have used the identity (2.8).

Making use of the integral representation

$$
H_{\mu}^{(1)}(k r) \mathrm{e}^{\mathrm{i} \mu \theta}=\frac{(-\mathrm{i})^{\mu+1}}{\pi} \int_{-\infty}^{\infty} \frac{\mathrm{e}^{-k \gamma(t)|y|}}{\gamma(t)} \mathrm{e}^{\mathrm{i} k x t} \mathrm{e}^{\mathrm{i} \mu \operatorname{sgn}(y) \arccos t} \mathrm{~d} t,
$$

in which $x=r \cos \theta, y=r \sin \theta$ and $\gamma(t)$ is defined for real $t$ by

$$
\gamma(t)= \begin{cases}-\mathrm{i} \sqrt{1-t^{2}}, & |t| \leq 1 \\ \sqrt{t^{2}-1}, & |t|>1\end{cases}
$$


and the Poisson summation formula, it turns out that the far field consists of a set of plane waves propagating in the directions $\theta= \pm \chi_{m}$ :

$$
\phi \sim \phi^{\mathrm{In}}+\frac{\pi \mathrm{i}}{k R} f_{0}(z) \sum_{m \in \mathcal{M}} \frac{1}{\sin \chi_{m}} \mathrm{e}^{\mathrm{i} k r \cos \left(\theta \mp \chi_{m}\right)} \sum_{\mu=-\infty}^{\infty} A_{0 \mu} \mathrm{e}^{ \pm \mathrm{i} \mu \chi_{m}}
$$

It is implicit in all the above that $\sin \chi_{m} \neq 0$ for any $m$. If $\sin \chi_{m}=0$ then we have the situation where one of the scattered plane waves propagates along the array. We will not consider this resonant case here but refer to Thompson \& Linton (2006) where this special case is investigated in detail. From (6.4) the amplitudes of the scattered waves for each scattering angle $\pm \chi_{m}$ are given in terms of the coefficients $A_{0 \mu}$ by

$$
A_{m}^{ \pm}=\frac{\pi \mathrm{i}}{k R} \frac{1}{\sin \chi_{m}} \sum_{\mu=-\infty}^{\infty} A_{0 \mu} \mathrm{e}^{ \pm \mathrm{i} \mu \chi_{m}}
$$

Note that the primary reflection and transmission coefficients are given by $A_{0}^{-}$and $1+A_{0}^{+}$, respectively.

\subsection{Semi-infinite array}

For the semi-infinite array, our derivation relies heavily on that of Linton \& Martin (2004). Their setting is simpler, however. In particular, it does not support RayleighBloch waves.

Making use of (6.3), the total field is

$$
\begin{aligned}
\phi=\phi^{\mathrm{In}}+\sum_{m=0}^{\infty} f_{m}(z) \sum_{j=0}^{\infty} \sum_{\mu=-\infty}^{\infty}\left(P_{j} A_{m \mu}+Q_{j} \mathcal{A}_{m \mu}+Z_{m \mu}^{j}\right) K_{\nu}\left(k_{m} r_{j}\right) \mathrm{e}^{\mathrm{i} \mu \theta_{j}} \\
\sim \phi^{\mathrm{In}}+\frac{1}{2} f_{0}(z) \sum_{j=0}^{\infty} \sum_{\mu=-\infty}^{\infty}\left(P_{j} A_{0 \mu}+Q_{j} \mathcal{A}_{m \mu}+Z_{0 \mu}^{j}\right) \\
\times \int_{-\infty}^{\infty} \frac{\mathrm{e}^{-k \gamma(t)|y|}}{\gamma(t)} \mathrm{e}^{\mathrm{i} k(x-j R) t} \mathrm{e}^{\mathrm{i} \mu \operatorname{sgn}(y) \arccos t} \mathrm{~d} t
\end{aligned}
$$

as $k r \rightarrow \infty$, away from the array axis $y=0$.

We begin with the asymptotics of the term

$$
\sum_{j=0}^{\infty} P_{j} \int_{-\infty}^{\infty} \frac{\mathrm{e}^{-k \gamma(t)|y|}}{\gamma(t)} \mathrm{e}^{\mathrm{i} k(x-j R) t} \mathrm{e}^{\mathrm{i} \mu \operatorname{sgn}(y) \arccos t} \mathrm{~d} t .
$$

Making use of the half-range summation formula (Linton \& Martin 2004, eq. (B.2)),

$$
\sum_{m=0}^{\infty} \int_{-\infty}^{\infty} f(u) \mathrm{e}^{-\mathrm{i} m u} \mathrm{~d} u=f_{-\infty}^{\infty} \frac{f(u)}{1-\mathrm{e}^{-\mathrm{i} u}} \mathrm{~d} u+\pi \sum_{m=-\infty}^{\infty} f(2 m \pi)=f_{-\infty}^{\infty} \frac{f(u)}{1-\mathrm{e}^{-\mathrm{i} u}} \mathrm{~d} u
$$

to express the sum as a contour integral (where the notation $f$ means that the contour passes below the poles of the integrands) and writing

$$
\tilde{f}_{\mu}(t)=\mathrm{e}^{\mathrm{i} \mu \operatorname{sgn}(y) \arccos t}
$$


for ease of notation, we obtain

$$
\begin{aligned}
\sum_{j=0}^{\infty} P_{j} \int_{-\infty}^{\infty} \frac{\mathrm{e}^{-k \gamma(t)|y|}}{\gamma(t)} \mathrm{e}^{\mathrm{i} k(x-j R) t} \tilde{f}_{\mu}(t) \mathrm{d} t=f_{-\infty}^{\infty} \frac{\mathrm{e}^{-k \gamma(t)|y|} \mathrm{e}^{\mathrm{i} k x t} \tilde{f}_{\mu}(t)}{\gamma(t)\left(1-\mathrm{e}^{\mathrm{i} R(\psi-k t)}\right)} \mathrm{d} t \\
\sim \frac{\mathrm{i} \tilde{f}_{\mu}(\cos \theta) \mathrm{e}^{\mathrm{i}(k r-\pi / 4)}}{1-\mathrm{e}^{\mathrm{i} k R(\cos \chi-\cos \theta)}} \sqrt{\frac{2 \pi}{k r}}+2 \pi \mathrm{i} \sum_{\substack{m \in \mathcal{M} \\
\chi_{m}>\theta}} \frac{\tilde{f}_{\mu}\left(\psi_{m} / k\right) \mathrm{e}^{\mathrm{i} k r \cos \left(|\theta|-\chi_{m}\right)}}{k R \sin \chi_{m}}
\end{aligned}
$$

as $k r \rightarrow \infty$ where we have made use of the asymptotics of the contour integral given by (Linton \& Martin 2004, eq. (F.1)).

Similarly, for the term involving the coefficient of the Rayleigh-Bloch wave, we obtain

$$
\sum_{j=0}^{\infty} Q_{j} \int_{-\infty}^{\infty} \frac{\mathrm{e}^{-k \gamma(t)|y|}}{\gamma(t)} \mathrm{e}^{\mathrm{i} k(x-j R) t} \tilde{f}_{\mu}(t) \mathrm{d} t \sim \frac{\mathrm{i} \tilde{f}_{\mu}(\cos \theta) \mathrm{e}^{\mathrm{i}(k r-\pi / 4)}}{1-\mathrm{e}^{\mathrm{i} R(\beta-k \cos \theta)}} \sqrt{\frac{2 \pi}{k r}}
$$

as $k r \rightarrow \infty$ where the sum over the scattering angles vanishes in the derivation since $\beta>k$.

For the sum over $j$ involving $Z_{0 \mu}^{j}$,

$$
\sum_{j=0}^{\infty} Z_{0 \mu}^{j} \int_{-\infty}^{\infty} \frac{\mathrm{e}^{-k \gamma(t)|y|}}{\gamma(t)} \mathrm{e}^{\mathrm{i} k(x-j R) t} \tilde{f}_{\mu}(t) \mathrm{d} t
$$

we take the sum inside the integral (the assumption $Z_{0 \mu}^{j} \rightarrow 0$ as $j \rightarrow \infty$ ensures that $\left|Z_{0 \mu}^{j} \mathrm{e}^{-\mathrm{i} j k R t}\right|$ becomes small enough for large $j$ ) and use stationary phase (in exactly the same way as for (6.6), except that there are now no poles to worry about). In summary, we find that

$$
\phi \sim \phi^{\mathrm{In}}+f_{0}(z) \tilde{H}(k r) g(\theta)+\frac{\pi \mathrm{i} f_{0}(z)}{k R} \sum_{\substack{m \in \mathcal{M} \\ \chi_{m}>|\theta|}} \frac{\mathrm{e}^{\mathrm{i} k r \cos \left(|\theta|-\chi_{m}\right)}}{\sin \chi_{m}} \sum_{\mu=-\infty}^{\infty} A_{0 \mu} \mathrm{e}^{\mathrm{i} \mu \operatorname{sgn}(\theta) \chi_{m}}
$$

where $\tilde{H}(k r)=\sqrt{\pi / 2 k r} \exp (\mathrm{i}(k r-\pi / 4))$ and

$$
g(\theta)=\mathrm{i} \sum_{\mu=-\infty}^{\infty} \mathrm{e}^{\mathrm{i} \mu \theta}\left(\frac{A_{0 \mu}}{1-\mathrm{e}^{\mathrm{i} k R(\cos \chi-\cos \theta)}}+\frac{\mathcal{A}_{0 \mu}}{1-\mathrm{e}^{\mathrm{i} R(\beta-k \cos \theta)}}+\sum_{j=0}^{\infty} Z_{0 \mu}^{j} \mathrm{e}^{-\mathrm{i} j k R \cos \theta}\right) .
$$

The diffracted field takes the form of a circular wave of 'amplitude' $g(\theta)$ plus a sum of plane waves which propagate in the same directions as for the infinite-grating case. However, unlike in the infinite-array problem, the plane waves do not exist everywhere and the wave making an angle $\chi_{m}\left(\right.$ resp. $\left.-\chi_{m}\right)$ with the $x$-axis is not found in the sector $0<\theta<\chi_{m}$ (resp. $0>\theta>-\chi_{m}$ ).

The coefficients $Z_{0 \mu}^{j}$ and the Rayleigh-Bloch coefficient $\mathcal{A}_{0 \mu}$ affect only the circular wave. The plane wave field is determined entirely from the solution of the infinite-grating problem; in fact, where the plane waves exist, their amplitude is precisely as in the infinite-grating problem.

The amplitude $g(\theta)$ of the circular wave becomes infinite as $\theta$ approaches one of the so-called shadow boundaries $|\theta|=\chi_{p}$. This is because, in performing the steepest descent analysis, it was explicitly assumed that $|\theta| \neq \chi_{p}$. Uniform asymptotics valid as $\theta \rightarrow \chi_{p}$ 
can be derived. It turns out (cf. Linton \& Martin 2004, eq. (F.2)) that the term

$$
\frac{\sqrt{\pi} \mathrm{e}^{\mathrm{i}(k r-\pi / 4)}}{\sqrt{2 k r} k R \sin \left(\left(|\theta|-\chi_{p}\right) / 2\right) \sin \chi_{p}} \tilde{f}_{\mu}\left(\psi_{p} / k\right)\left(1+2 \mathrm{i} \zeta_{p} \mathrm{e}^{-\mathrm{i} \zeta_{p}^{2}} F\left(\zeta_{p}\right)\right)
$$

must be added to the right-hand side of (6.6) where $\zeta_{p}=\sqrt{2 k r} \sin \left(|| \theta\left|-\chi_{p}\right| / 2\right)$ and

$$
F(v)=\int_{v}^{\infty} \mathrm{e}^{\mathrm{i} u^{2}} \mathrm{~d} u \quad(0<\arg u<\pi / 2 \text { as } u \rightarrow \infty)
$$

is a Fresnel integral. Therefore, near the shadow boundaries, the term

$$
\tilde{g}(r, \theta)=\frac{1+2 \mathrm{i} \zeta_{p} \mathrm{e}^{-\mathrm{i} \zeta_{p}^{2}} F\left(\zeta_{p}\right)}{2 k R \sin \left(\left(|\theta|-\chi_{p}\right) / 2\right) \sin \chi_{p}} \sum_{\mu=-\infty}^{\infty} A_{0 \mu} \mathrm{e}^{\mathrm{i} \mu \operatorname{sgn}(\theta) \chi_{p}}
$$

must be added to (6.10) so that the amplitude of the circular wave is given by $g(\theta)+\tilde{g}(r, \theta)$. The combination $g+\tilde{g}$ is bounded as $\theta \rightarrow \chi_{p}$ for any $r$, but the limit is different from each side. However, since $F(0)=\frac{1}{2} \sqrt{\pi} \exp (\mathrm{i} \pi / 4)$, the discontinuity in $g+\tilde{g}$ as $\theta$ passes through $\chi_{p}$ exactly cancels the extra plane-wave contribution that appears in (6.6) as the shadow boundary is crossed, thus ensuring that the far field is continuous.

\section{The efficient computation of the Schlömilch series}

The values of the Schlömilch series $\sigma_{\nu}^{n}$ (cf. (3.2)), $\tilde{\varsigma}_{\nu}^{n l}, \hat{\varsigma}_{\nu}^{n l}$ (cf. (5.2)) and $\varsigma_{\nu}^{n l}$ (cf. (5.9)) appearing in the systems of equations for the coefficients of the scattered wavefield of the bodies cannot be computed straightforwardly for $n=0$. This is because the sums are not absolutely convergent due to the slow decay of the modified Bessel function of the second kind for large imaginary argument (The terms in the sum decay like $j^{-1 / 2} \mathrm{e}^{\mathrm{i} j \theta}$ for some $\theta$ ). The calculation of the constants for $n \neq 0$ is easy, however, since the modified Bessel function of the second kind decays exponentially for large real argument.

The efficient computation of the slowly-convergent series appearing in the infinite-array calculation, $\sigma_{\nu}^{0}$, which are often referred to as lattice sums, is discussed in detail in Peter et al. (2006) using the same notation as is used here. It is based on the results of Linton (1998).

Therefore, we focus on the efficient computation of $\tilde{\varsigma}_{\nu}^{0 l}, \hat{\varsigma}_{\nu}^{0 l}$ and $\varsigma_{\nu}^{0 l}$. First, note that

$$
\begin{aligned}
\hat{\varsigma}_{\nu}^{n l} & =\sum_{j=1}^{\infty} K_{\nu}\left(k_{n} j R\right) \mathrm{e}^{-\mathrm{i} j R k \cos \chi}-\sum_{j=1}^{l} K_{\nu}\left(k_{n} j R\right) \mathrm{e}^{-\mathrm{i} j R k \cos \chi} \\
\hat{\varsigma}_{\nu}^{n l} & =\sum_{j=1}^{\infty} K_{\nu}\left(k_{n} j R\right) \mathrm{e}^{-\mathrm{i} j R \beta}-\sum_{j=1}^{l} K_{\nu}\left(k_{n} j R\right) \mathrm{e}^{-\mathrm{i} j R \beta} \\
\varsigma_{\nu}^{n l} & =(-1)^{\nu} \sum_{j=1}^{\infty} K_{\nu}\left(k_{n} j R\right) \mathrm{e}^{\mathrm{i} j R \beta}-(-1)^{\nu} \sum_{j=1}^{N-l} K_{\nu}\left(k_{n} j R\right) \mathrm{e}^{\mathrm{i} j R \beta} .
\end{aligned}
$$

It therefore suffices to discuss the efficient computation of the series

$$
\varsigma_{\nu}^{ \pm}(u)=\sum_{j=1}^{\infty} K_{\nu}(-\mathrm{i} k j R) \mathrm{e}^{ \pm \mathrm{i} j R u}=\frac{\pi \mathrm{i}^{\nu+1}}{2} \sum_{j=1}^{\infty} H_{\nu}^{(1)}(k j R) \mathrm{e}^{ \pm \mathrm{i} j R u}
$$

where we have used (2.8). An efficient way of calculating these series has only been found recently (Linton 2006). Since it is rather involved we refer to Linton (2006) for details. 


\section{Numerical simulations}

We present numerical calculations for rigid bottom-mounted circular cylinders, fixed docks and ice floes. The ice floes are taken as floating flexible plates of shallow draft as modelled by Meylan (2002).

The diffraction transfer matrices of rigid bottom-mounted circular cylinders are particularly simple owing to the axisymmetry. In fact, they are diagonal with diagonal elements

$$
(\mathbf{B})_{p p}=-I_{p}^{\prime}\left(k_{0} a\right) / K_{p}^{\prime}\left(k_{0} a\right) .
$$

There are no decaying modes if the ambient incident wave does not contain decaying modes (which is the case in our considerations). Details for the calculation of the diffraction transfer matrices for floating plates can be found in Peter et al. (2006).

The remainder of this section consists of three parts. We begin by investigating the convergence of the method for the case that a Rayleigh-Bloch wave is present. For this purpose, we consider the scattering by semi-infinite arrays of cylinders. In the second part, we investigate the existence of Rayleigh-Bloch waves for a more complicated structure than a cylinder, namely circular floating stiff plates, for two parameter sets. It turns out that in the cases considered, Rayleigh-Bloch waves do not exist. In the third part of this section, the scattering by semi-infinite arrays of floating flexible plates is investigated and compared to that of an analogous array of docks as well as the corresponding infinite array.

In all calculations presented, the water depth is $d=1 / 2$ and the ambient incident wavefield is of unit amplitude (in displacement) and travels in a direction making an angle of $\chi=\pi / 5$ with the $x$-axis.

\subsection{Cylinders}

We investigate the convergence of both the subtraction method and the projection method for a semi-infinite array of bottom-mounted circular cylinders. We choose the wavenumber $k=1$, the body spacing $R=2$ and we consider cylinders with two different radii, $a_{1}=1 / 2$ and $a_{2}=3 / 4$. Note that there is only one scattering angle in this case, namely the angle of the ambient incident wave $\chi=\pi / 5$. For both cases, it is sufficient to use eleven modes in the calculations.

For the cylinders of radius $a_{1}=1 / 2$, the Rayleigh-Bloch wavenumber can be calculated as $\beta_{1}=1.013398$ and we have $A_{0}^{-}=0.060267-0.175943 \mathrm{i}$ and $A_{0}^{+}=-0.070466+$ 0.318400 i. For $a_{2}=3 / 4$, we have $\beta_{2}=1.025629$ and $A_{0}^{-}=0.327177-0.467147 \mathrm{i}$ and $A_{0}^{+}=-0.327185+0.471221 \mathrm{i}$. The potential of the Rayleigh-Bloch wave associated with the zeroth cylinder is plotted at the cylinder boundary in figure 2 for both radii, i.e. we plot $\phi_{0}^{\mathrm{RB}}\left(a_{k}, \theta\right), k=1,2$, where

$$
\phi_{0}^{\mathrm{RB}}(r, \theta)=\sum_{\mu=-\infty}^{\infty} v_{0 \mu} K_{\mu}\left(k_{0} r\right) \mathrm{e}^{\mathrm{i} \mu \theta} .
$$

This visualizes the numerical results obtained for $v$.

Using this data, the complex constant $c$ (cf. $§ 5.2)$ is determined using the least-squares fit (that is, finding the complex $c$ such that $Z^{N}$ is as small as possible) as well the projection method (where it is calculated a-posteriori as $c=Q_{-N}\left(\mathbf{z}_{N} \mid \mathbf{v}\right)$ ). The real and imaginary parts of $c$ obtained using $N$ cylinders in the calculation are plotted in figure 3 for increasing $N$. Some numerical values are given in table 1 . It can be seen that the method is only slowly convergent; however, reasonable accuracy, which will be sufficient for most applications, is already obtained for a small number of bodies. Also, the two methods give virtually identical accuracy (it should be noted that the subtraction method 

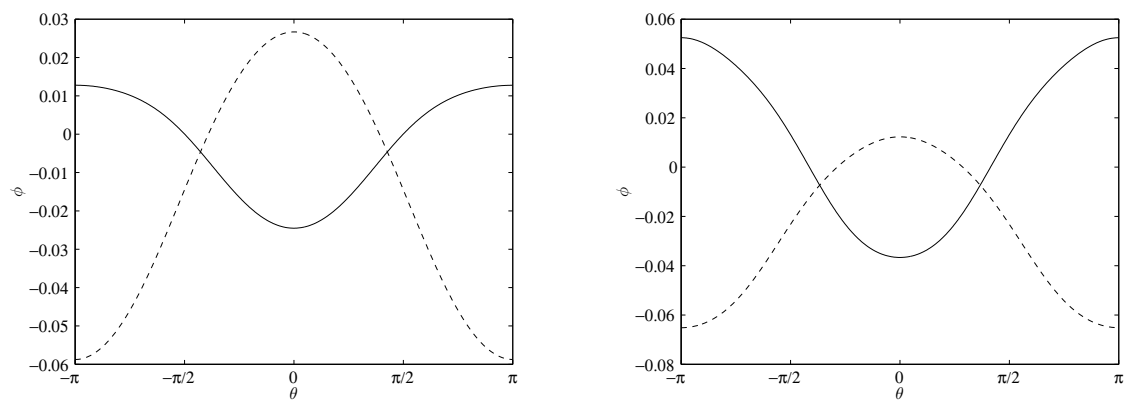

FiguRE 2. Real part (solid line) and imaginary part (dashed line) of the potential of the Rayleigh-Bloch wave associated with the zeroth cylinder at the cylinder boundary, $\phi_{0}^{\mathrm{RB}}\left(a_{k}, \theta\right)$ for $a_{1}=1 / 2$ (left) and $a_{2}=3 / 4$ (right), also cf. (8.2).
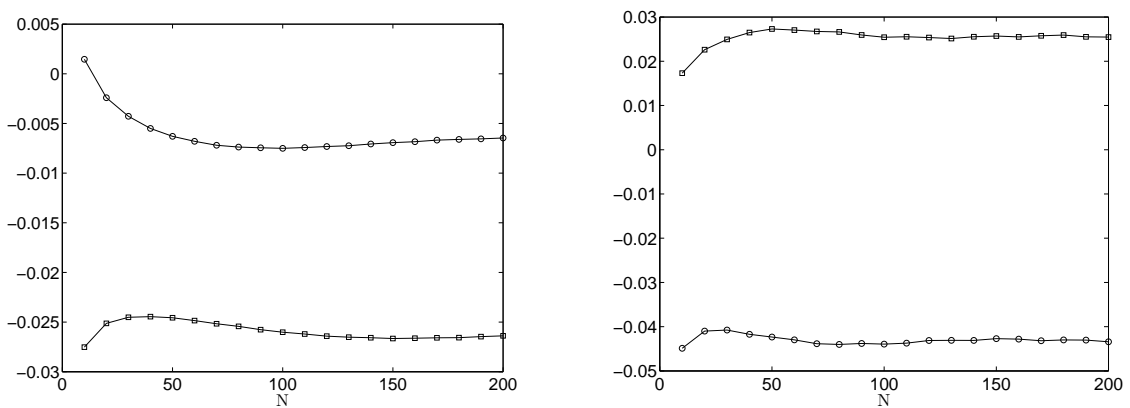

Figure 3. Real ( $\square$ ) and imaginary (o) part of $c$ for increasing number of cylinders calculated with the projection method for $a_{1}=1 / 2$ (left) and $a_{2}=3 / 4$ (right).

$\begin{array}{ccc}N & c \text { (proj. meth.) } & c \text { (subtr. meth.) } \\ 50 & -0.024565-0.006299 \mathrm{i} & -0.024560-0.006261 \mathrm{i} \\ 100 & -0.026018-0.007509 \mathrm{i} & -0.026001-0.007507 \mathrm{i} \\ 150 & -0.026659-0.006938 \mathrm{i} & -0.026656-0.006949 \mathrm{i} \\ 200 & -0.026386-0.006464 \mathrm{i} & -0.026395-0.006467 \mathrm{i} \\ & c \text { (proj. meth.) } & c \text { (subtr. meth.) } \\ N & +0.027302-0.042334 \mathrm{i} & +0.027247-0.042274 \mathrm{i} \\ 50 & +0.0258-0.043948 & +0.025495-0.043968 \\ 100 & +0.025438-0.043948 \mathrm{i} \\ 150 & +0.025712-0.042708 \mathrm{i} & +0.025668-0.042711 \mathrm{i} \\ 200 & +0.025468-0.043419 \mathrm{i} & +0.025514-0.043400 \mathrm{i}\end{array}$

TABLE 1. The constant $c$ calculated with the projection method and the subtraction method using $N$ cylinders for $a_{1}=1 / 2$ (above) and $a_{2}=3 / 4$ (below).

is less numerically efficient because the least-squares approach can require considerable computation time).

In figure 4 , the total wavefield and the scattered wavefield only are plotted. The circular wave radiating from the edge and the plane waves (note that there are only two in each setting, propagating in the directions $\pm \chi$ ) can be clearly seen. Note also, that the reflected wave is of much greater amplitude for the cylinders with greater radius. Figure 5 shows the Rayleigh-Bloch wave as it travels down the corresponding infinite array, i.e. we plot the surface displacement corresponding to $c \phi^{\mathrm{RB}}$. The wavenumber of this wave is larger than the ambient wave and the wave decays quickly away from the array. 

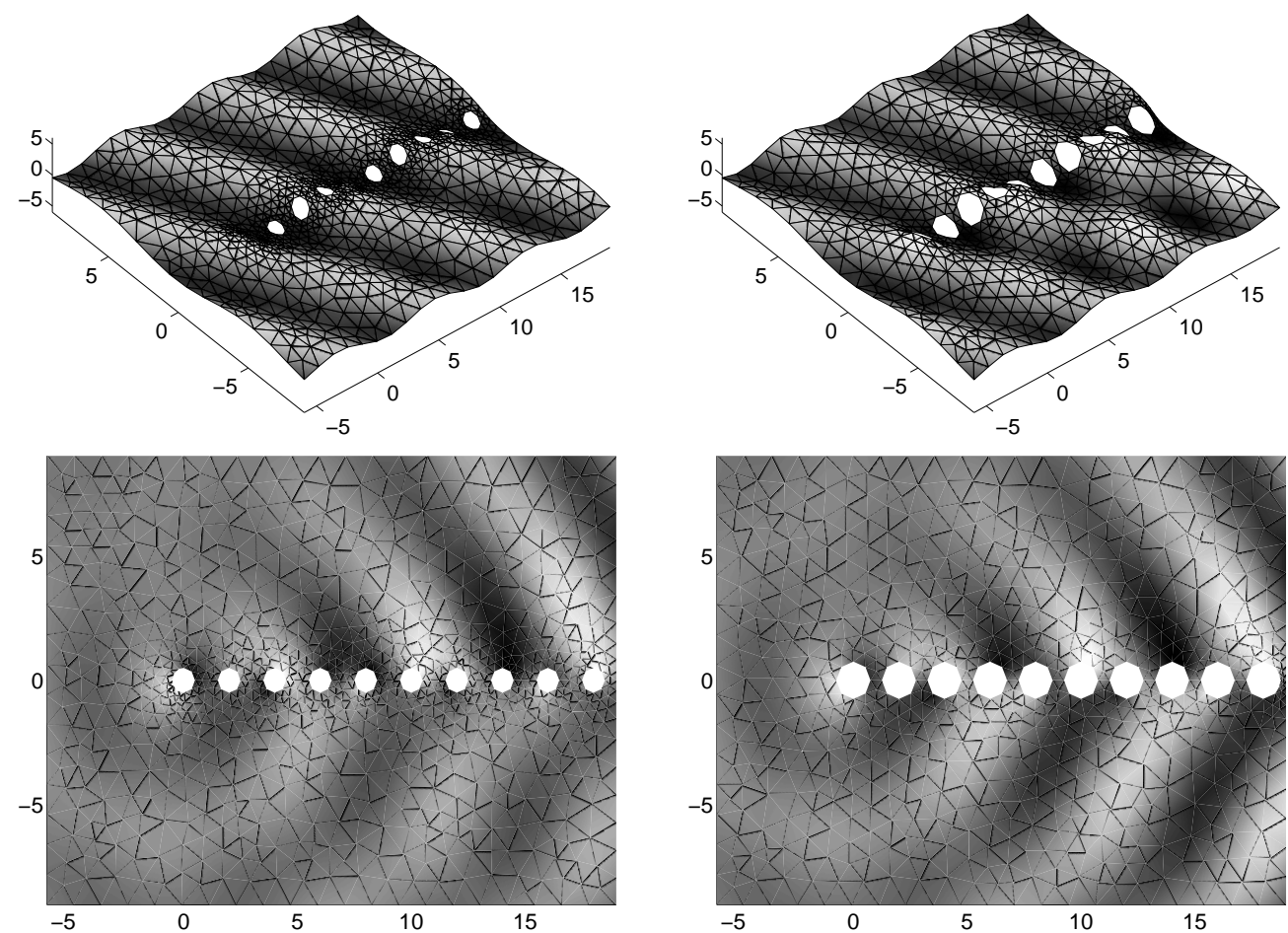

FIGURE 4. Total wavefield (top) and scattered wavefield only (bottom) of the solution for the semi-infinite array of cylinders with radius $a_{1}=1 / 2$ (left) and $a_{2}=3 / 4$ (right).

In figure 6 , the absolute value of the amplitude of the circular wave, $|g(\theta)+\tilde{g}(r, \theta)|$ is plotted for $r=2 \pi$ (i.e. $r$ is one wavelength of the ambient wave). The discontinuities in the amplitude at the shadow boundaries, $\theta= \pm \chi$, and the array axis, $\theta=0$, can be clearly seen.

\subsection{Rayleigh-Bloch waves for arrays of circular stiff plates}

We briefly present some results concerning the existence of Rayleigh-Bloch waves for arrays of circular stiff plates where the plates are modelled as floating stiff plates of shallow draft as in Peter et al. (2006). The circular stiff plate is one of the most straightforward generalizations of a circular cylinder and it is therefore an obvious starting point to look for Rayleigh-Bloch waves in the general three-dimensional case. Instead of using the solution method described in Peter et al. (2006) (which is based on the finite element method), we use the matched-asymptotic-expansion method of Peter et al. (2004) to calculate the diffraction transfer matrix of a single circular stiff plate. This method is much more accurate but it is limited to circular plates. We take the same parameters as in the previous subsection, i.e. wavenumber $k=1$, body spacing $R=2$ and radius $a=3 / 4$ and we consider the two cases where the plates have (non-dimensional) mass $\tilde{\gamma}=0$ and $\tilde{\gamma} \rightarrow \infty$ (the latter of which corresponds to a fixed dock and allows further simplification), respectively. For these calculations, we again use eleven propagating modes but also eleven evanescent modes and one root of the dispersion relation (2.4).

In figure 7, the determinant of the matrix $\left(\mathbf{I}-\mathbf{B}^{\mathrm{t}} \mathbf{S}^{\mathrm{inf}}(\beta)\right.$ ) (cf. (4.3)) is plotted over $\beta \in(k, \pi / R]$. Recall that this determinant has to have a zero if there exists a RayleighBloch wave. As can be seen, the determinants do not have zeros in the critical interval, 

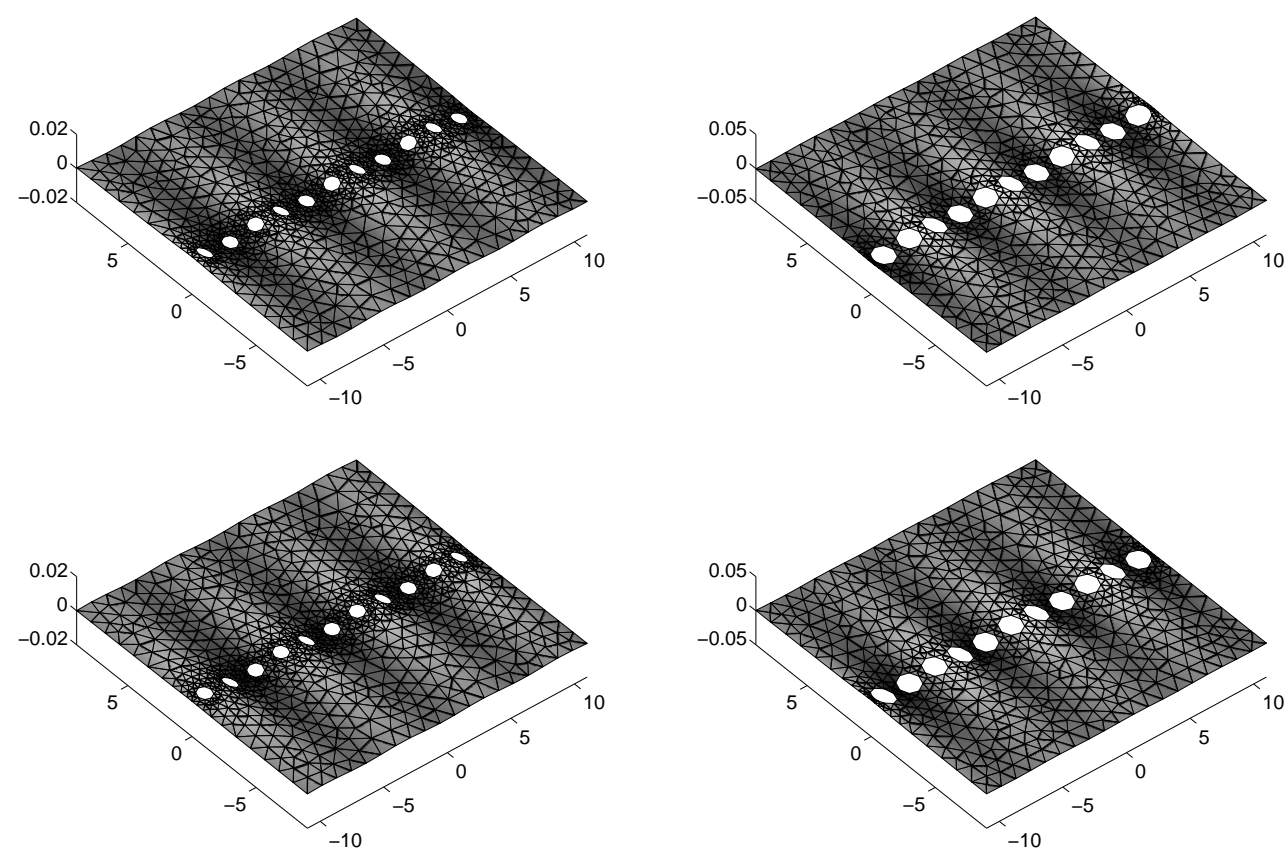

FIGURE 5. Rayleigh-Bloch wave excited in the semi-infinite array of cylinders with radius $a_{1}=1 / 2$ (left) and $a_{2}=3 / 4$ (right). Top: real part; bottom: imaginary part.
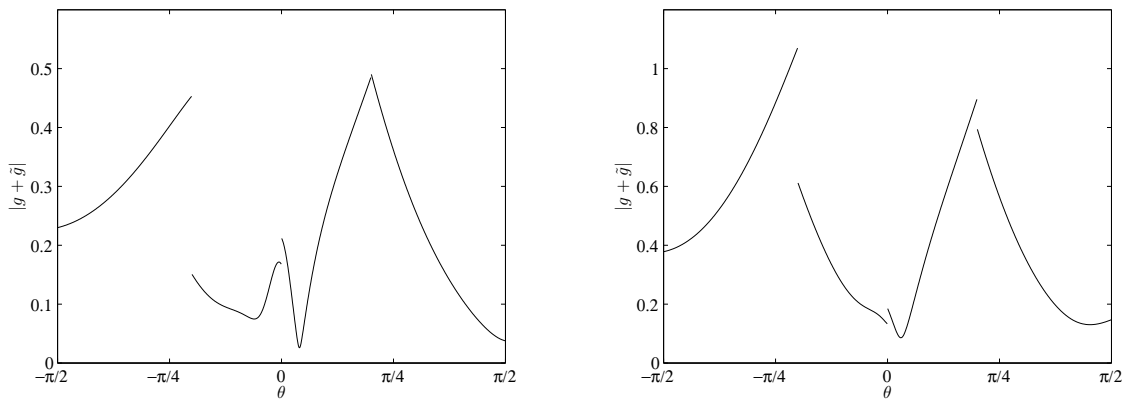

Figure 6. Absolute value of the amplitude of the circular wave, $|g(\theta)+\tilde{g}(r, \theta)|$, for $r=2 \pi$ and $a_{1}=1 / 2$ (left) and $a_{2}=3 / 4$ (right).

which implies that no Rayleigh-Bloch wave is supported. It is also observed that the imaginary part of the determinant is almost zero if $\tilde{\gamma}=0$ (figure 7, left) and the ratio of the real part and the imaginary part is a constant for the case of a dock (figure 7 , right). Similar curves are obtained for radius $a=1 / 2$.

Unlike in the two-dimensional case (corresponding to Helmholtz scattering), where Rayleigh-Bloch waves have been proven to exist in a large number of situations, it appears that this is not the case for the general three-dimensional problem. However, it needs to be noted that the above results do not allow any general conclusions and it seems likely that Rayleigh-Bloch waves do exist for some special structures even in the general case.

\subsection{Docks and ice floes}

We present results for fixed docks and floating elastic plates. For comparability, we use the same parameter set as was used by Peter et al. (2006) for the infinite-array problem, 

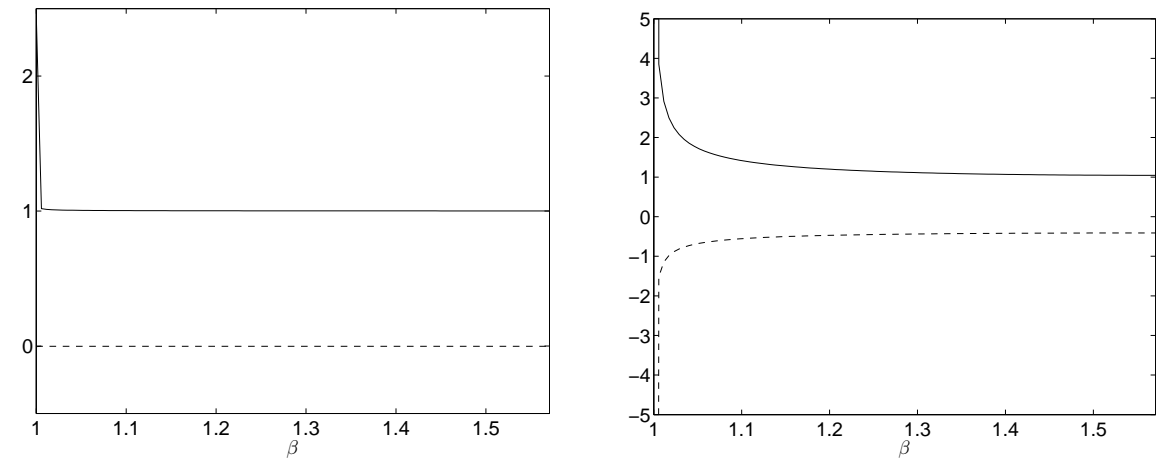

Figure 7. Real part (solid line) and imaginary part (dashed line) of the determinant of $\left(\mathbf{I}-\mathbf{B}^{\mathrm{t}} \mathbf{S}^{\mathrm{inf}}(\beta)\right)$ over plotted over $\beta$ for a circular stiff plate with non-dimensional mass $\tilde{\gamma}=0$ (left) and $\tilde{\gamma} \rightarrow \infty$ (right).

that is ambient wavelength $\lambda=1.5$ and spacing $R=4$. The bodies are square with side length 2 . The elastic plates are chosen to have non-dimensional stiffness and mass $\tilde{\beta}=\tilde{\gamma}=0.02$ (where the tildes have been introduced in order to avoid confusion with the Rayleigh-Bloch wavenumber).

In figure 8, it can be clearly seen that the docks scatter the waves much more strongly than the elastic plates. Moreover, the circular waves and the shadow boundaries are clearly apparent in the plots of the scattered wavefields. Further down the semi-infinite array, it can be seen that the scattered wavefield becomes more and more similar to that of the corresponding infinite array.

\section{Summary}

We have presented a solution to the scattering of water waves from a semi-infinite array of identical bodies of arbitrary geometry and scattering properties. Under certain simplifying assumptions our solution reduces to the problem of solving the two-dimensional Helmholtz equation. This solution has a range of applications outside water waves, for example acoustic or electromagnetic scattering. The solution method generalizes the results of Linton \& Martin (2004) who solved the two-dimensional Helmholtz equation for point scatterers. As well as extending the formulation to general bodies and waterwave scattering we have also shown how to include the effect of Rayleigh-Bloch waves in the formulation. These are bound waves, which exist for many geometries in the twodimensional Helmholtz-equation problem and they are excited in the semi-infinite problem. We have presented a range of solutions for various geometries focusing on scattering by cylinders in the simplest Helmholtz-equation case (where there are Rayleigh-Bloch waves) and on the highly non-trivial scattering by arrays of floating plates.

This research was supported by a Marsden Grant from the New Zealand government. The major part of this research was completed during a research stay of M.A.P. at the University of Auckland, supported by the University of Auckland. We would like to thank Richard Porter and Chris Linton for fruitful discussions and Garry Tee for suggestions on the manuscript.

\section{REFERENCES}

Abramowitz, M. \& Stegun, I. A., ed. 1970 Handbook of Mathematical Functions. Dover. 

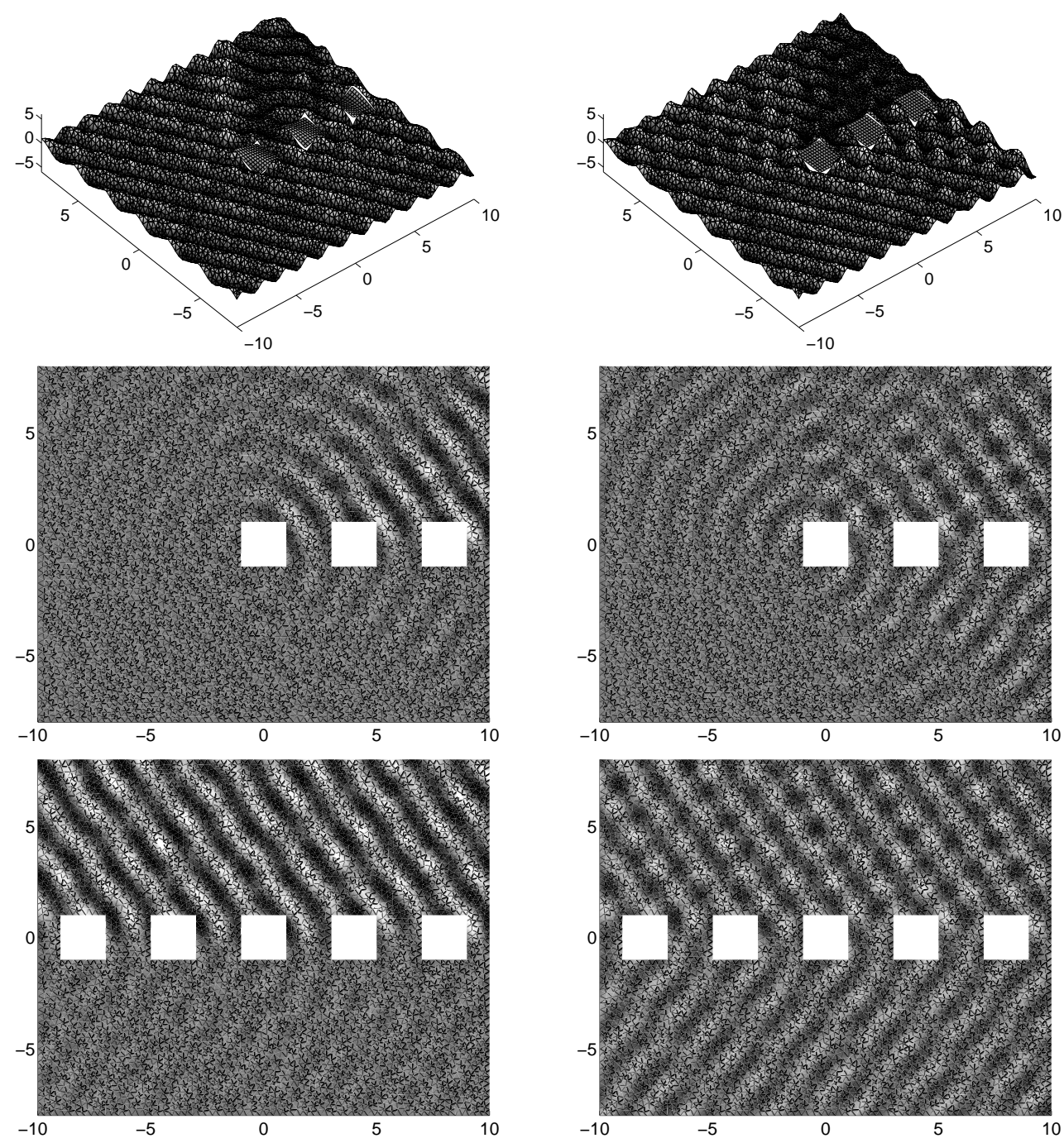

FIGURE 8. Solution to the scattering problem for an array of floating elastic plate with non-dimensional stiffness and mass $\tilde{\beta}=\tilde{\gamma}=0.02$ (left) and array for fixed docks (right). Top: total wavefield; middle: scattered wavefield; bottom: scattered wavefield of the corresponding infinite array.

Hills, N. L. \& Karp, S. N. 1965 Semi-infinite diffraction gratings I. Comm. Pure Appl. Math. 18, 203-233.

von Ignatowsky, W. 1914 Zur Theorie der Gitter. Ann. Phys. 44, 369-436.

Kagemoto, H. \& Yue, D. K. P. 1986 Interactions among multiple three-dimensional bodies in water waves: an exact algebraic method. J. Fluid Mech. 166, 189-209.

Linton, C. M. 1998 The Green's function for the two-dimensional Helmholtz equation in periodic domains. J. Engng. Math. 33, 377-402.

Linton, C. M. 2006 Schlömilch series that arise in diffraction theory and their efficient computation. J. Phys. A: Math. Gen. 39, 3325-3339.

Linton, C. M. \& Evans, D. V. 1993 The interaction of waves with a row of circular cylinders. J. Fluid Mech. 251, 687-708. 
Linton, C. M. \& Martin, P. A. 2004 Semi-infinite arrays of isotropic point scatterers. A unified approach. SIAM J. Appl. Math. 64 (3), 1035-1056.

Linton, C. M. \& McIver, M. 2002 The existence of Rayleigh-Bloch surface waves. J. Fluid Mech. 470, 85-90.

McIver, P. 2002 Wave interaction with arrays of structures. Appl. Ocean Res. 24, 121-126.

Meylan, M. H. 2002 Wave response of ice floes of arbitrary geometry. J. Geophys. Res. - Oceans 107 (C1), doi: 10.1029/2000JC000713.

Peter, M. A. \& Meylan, M. H. 2004 Infinite-depth interaction theory for arbitrary floating bodies applied to wave forcing of ice floes. J. Fluid Mech. 500, 145-167.

Peter, M. A., Meylan, M. H. \& Chung, H. 2004 Wave scattering by a circular elastic plate in water of finite depth: a closed form solution. IJOPE 14 (2), 81-85.

Peter, M. A., Meylan, M. H. \& Linton, C. M. 2006 Water-wave scattering by a periodic array of arbitrary bodies. J. Fluid Mech. 548, 237-256.

Porter, R. \& Evans, D. V. 1999 Rayleigh-Bloch surface waves along periodic gratings and their connection with trapped modes in waveguides. J. Fluid Mech. 386, 233-258.

Porter, R. \& Evans, D. V. 2005 Embedded Rayleigh-Bloch surface waves along periodic rectangular arrays. Wave Motion 43, 29-50.

Thompson, I. \& Linton, C. M. 2006 Resonant effects in scattering by periodic arrays. In Proc. 21st Int. Workshop on Water Waves and Floating Bodies, pp. 173-176.

Twersky, V. 1962 On scattering of waves by the infinite grating of circular cylinders. IRE Trans. on Antennas and Propagation 10, 737-765. 